\title{
An Improved Efficient Remote User Authentication Scheme in Multi-server Environment using Smart Card
}

\author{
Ruhul Amin \\ Haldia Institute of Technology \\ Haldia, West Bengal-721657, India
}

\author{
Tanmoy Maitra \\ Haldia Institute of Technology \\ Haldia, West Bengal-721657, India
}

\author{
Debasis Giri \\ Haldia Institute of Technology \\ Haldia, West Bengal-721657, India
}

\begin{abstract}
In a single server environment, one server is responsible for providing services to all the authorized remote users. However, the problem arises if a user wishes to access several network services. To overcome this weakness, various multi-server authentication schemes have been proposed. In 2012, Taygi et al. [1] proposed a scheme for multi-server environment. But it is found that their proposed scheme is insecure against user impersonation attack, server masquerading attack, collaboration attack between a valid user and a server, smart card stolen attack, password guessing attack and password change attack. Then we propose an improved scheme can overcome possible attacks and also provides better computational cost as well as communication cost than related schemes published earlier.
\end{abstract}

\section{Keywords:}

Attack, Authentication, Password, Smart Card

\section{INTRODUCTION}

In the client/server environment password-based authentication scheme with smart card is widely used in remote user authentication. Li et al. [2] first proposed authentication scheme for multi-server architecture based on neural networks. They had shown that their scheme allows users to choose the password freely. Lin et al. [3] found that Li et al.'s scheme takes long time on training neural networks and they proposed an improved scheme using ElGamal digital signature [4] and geometric properties on the Euclidean plane. Cao and Zhong [5] proved that there is impersonation attack and every users have to store large amount of public parameters in memory of smart card for authentication in Lin et al.'s scheme. Juang [6] proposed a scheme based on nonce, one-way hash function and symmetric cryptosystem, which is efficient with an additional trait of generating session key. Neverless, Ku et al. [7] proved that Juang's scheme is exposed to insider attack and fails to provide forward secrecy. Chang and Lee [8] proposed an improvement scheme over Juang's scheme and claimed that their scheme is able to resist stolen-verified attack, server spoofing attack, smart card loss attack, reply attack and provides mutual authentication and forward secrecy. Liao and Wang [9] proposed a dynamic ID-based remote user authentication scheme based on one way hash function. But Chen et al. [10] pointed out that Liao-Wang's scheme does not provide forward secrecy. Hsiang and Shih [11] found that Liao-Wang's scheme is insecure against insider attack, impersonation attack, server spoofing attack and shows inadequacy in providing mutual authentication. To remove these drawbacks, they also proposed an improved scheme. Though, Sood et al. [12] pointed out that Hsiang-Shih's improved scheme is insecured against reply attack, impersonation attack, stolen smart card attack. Tsai [13] suggested a nonce based scheme based on one way hash function. However, Zhu [14] proved that Tsai's scheme is vulnerable to denning-sacco [15] attack, server spoofing attack and does not provide perfect forward secrecy. To overcome these drawbacks, they proposed an improved scheme.

In 2012, Taygi et al. [1] proposed a scheme based on nonce and one way hash function. In this paper, it is demonstrated that this scheme is insecure against user impersonation attack, server masquerading attack, collaboration attack between a valid user and a server, smart card stolen attack, password guessing attack and password change attack. To overcome these weakness we propose the improvement of their scheme. In addition, proposed scheme also provides better computational cost as well as communication cost than the related scheme published earlier.

The remainder of this paper is organized as follows: Section 2 briefly reviews the Taygi et al.'s [1] scheme. Section 3 shows the brief description of attacks on Taygi et al.'s [1] scheme. Section 4 describes the proposed scheme which withstand the weaknesses of Taygi et al.'s [1] scheme. Section 5 describes cryptanalysis of proposed improved scheme and section 6 compares the performances of proposed scheme with previously published scheme. Conclusion of this paper appears in section 7. Finally References are given in section 8 .

\section{BRIEF REVIEW OF TAYGI ET AL.'S SCHEME}

This section presents briefly description of Taygi et al.'s [1] remote user authentication scheme in multi server environment using smart card. The notations used throughout this paper are summarized as follows:

$$
\begin{array}{lll}
\mathrm{RC} & \longrightarrow & \text { registration center } \\
U_{i} & \longrightarrow & i-t h \text { remote user } \\
I D_{i} & \longrightarrow & \text { identity of } U_{i}
\end{array}
$$

Their scheme consists of following four phases: Registration phase, Login phase, Authentication phase and Password Change phase.

\subsection{Registration Phase}

This phase is divided into two sub-phases: Server Registration phase and User Registration phase.

\subsubsection{Server Registration Phase}

In this phase, $S_{j}$ selects $S I D_{j}$ and submits it to RC over a secure channel. After receiving it, RC computes the server secret parameter $S S_{j}=\left(g^{h\left(S I D_{j}, h(x)\right)} \bmod p\right) \oplus h(d)$ and sends $\left\{S S_{j}, h(d)\right\}$ to $S_{j}$ over a secure channel. 


$\begin{array}{lll}P W_{i} & \longrightarrow & \text { password chosen by } U_{i} \\ P W_{i}^{*} & \longrightarrow & \text { password guessed by an adversary } \\ S_{j} & \longrightarrow & j-t h \text { authentication server }(1 \leq j \leq n) \\ S I D_{j} & \longrightarrow & \text { identity of } S_{j} \\ x & \longrightarrow & \text { secret key of RC } \\ d & \longrightarrow & \text { secret number of RC } \\ p & \longrightarrow & \text { large prime number } \\ g & \longrightarrow & \text { primitive element over } G F(p) \\ h(.) & \longrightarrow & \text { cryptographic one way hash function } \\ \oplus & \longrightarrow & \text { bitwise XOR operation } \\ S K e y_{i j} & \longrightarrow & \text { session key shared between } U_{i} \text { and } S_{j} \\ N_{1} & \longrightarrow & \text { random nonce generated by } U_{i} \\ k & \longrightarrow & \text { Secret number chosen by } U_{i} \\ N_{2} & \longrightarrow & \text { random nonce generated by } S_{j} \\ n_{i} & \longrightarrow & \text { random nonce generated by RC for each user } \\ w_{j} & \longrightarrow & \text { random nonce generated by RC for each server }\end{array}$

\subsubsection{User Registration Phase}

$U_{i}$ selects $I D_{i}$ and $P W_{i}$, computes $h\left(P W_{i}\right)$ and submits $\left\{I D_{i}, h\left(P W_{i}\right)\right\}$ to $\mathrm{RC}$ over a secure channel. After receiving registration request, RC computes $x_{i}=\left(g^{h\left(P W_{i}\right)} \bmod p\right) \oplus$ $h(x), y_{i}=h\left(I D_{i}, h(d)\right), z_{i}=y_{i} \oplus h\left(P W_{i}\right)$ and issues a smart card for $U_{i}$ by storing $\left\{x_{i}, y_{i}, z_{i}, p, g, h(\cdot)\right\}$ for $(i=1$ to $n)$ into memory of smart card.

\subsection{Login Phase}

$U_{i}$ inserts his/her smart card to the card reader and then supply $I D_{i}$ and $P W_{i}$. The smart card computes $z_{i}^{\prime}=y_{i} \oplus h\left(P W_{i}^{\prime}\right)$ and checks whether computed $z_{i}^{\prime}$ equals stored $z_{i}$ or not. If equal, smart card generates a random nonce $N_{1}$, computes $a_{i}$ $=g^{y_{i}} \bmod p, b_{i}=a_{i}{ }^{y_{i} \times N_{1}} \bmod p, c_{i}=a_{i}{ }^{h\left(P W_{i}\right) \times N_{1}} \bmod p$, $d_{i}=g^{h\left(P W_{i}\right)} \bmod p, Q_{j}=g^{h\left(S I D_{j},\left(x_{i} \oplus d_{i}\right)\right)} \bmod p, e_{i}=$ $\left(h\left(P W_{i}\right)+y_{i} \times h\left(I D_{i}, a_{i}, b_{i}, c_{i}, d_{i}, N_{1}, Q_{j}\right)\right) \bmod (p-1)$ and sends the login request message $\left\{I D_{i}, S I D_{j}, d_{i}, e_{i}, N_{1}\right\}$ to $S_{j}$.

\subsection{Authentication phase}

After receiving the login request message $\left\{I D_{i}, S I D_{j}, d_{i}\right.$, $\left.e_{i}, N_{1}\right\}, S_{j}$ first checks the validity of $I D_{i}$. If valid, $S_{j}$ computes $y_{i}=h\left(I D_{i}, h(d)\right), a_{i}=g^{y_{i}} \bmod p, b_{i}=$ $a_{i}{ }^{y_{i} \times N_{1}} \bmod p, c_{i}=d_{i}{ }^{{ }_{i} \times N_{1}} \bmod p, Q_{j}=S S_{j} \oplus h(d)$ and checks whether $g^{e_{i}}=d_{i} \times a_{i}{ }^{h\left(I D_{i}, a_{i}, b_{i}, c_{i}, d_{i}, N_{1}, Q_{j}\right)} \bmod p$ is true. If it is true, $S_{j}$ further checks whether $a_{i}{ }^{e_{i} \times N_{1}}=$ $c_{i} \times b_{i}^{h\left(I D_{i}, a_{i}, b_{i}, c_{i}, d_{i}, N_{1}, Q_{j}\right)} \bmod p$ is true. If it holds good, $S_{j}$ generates a nonce $N_{2}$, computes $X_{1}=y_{i} \oplus N_{1} \oplus N_{2}$, $X_{2}=Q_{j}{ }^{N_{2}} \bmod p$ and sends the message $\left\{I D_{i}, X_{1}, X_{2}\right\}$ to $U_{i}$. After getting the message $\left\{I D_{i}, X_{1}, X_{2}\right\}$ from $S_{j}$, $U_{i}$ computes $N_{2}=y_{i} \oplus X_{1} \oplus N_{1}, X_{2}^{\prime}=Q_{j}{ }^{N_{2}} \bmod p$ and checks whether $X_{2}$ and $X_{2}^{\prime}$ are equal. If equality holds, $S_{j}$ is authentic, otherwise terminate the session. Subsequently, $U_{i}$ computes $X_{3}=Q_{j}{ }^{N_{1} \times N_{2}} \bmod p$ and sends $\left\{I D_{i}, X_{3}\right\}$ to $S_{j}$. Once the message $\left\{I D_{i}, X_{3}\right\}$ is received, $S_{j}$ computes $X_{3}^{\prime}=$ $Q_{j}^{N_{1} \times N_{2}} \bmod p$ and checks whether $X_{3}$ and $X_{3}^{\prime}$ are equal. If equality holds, mutual authentication is achieved. Both the parties agree upon a common shared session key $S K e y_{i j}=$ $h\left(I D_{i}, S I D_{j}, Q_{j}, N_{1}, N_{2}\right)$

\subsection{Password Change phase}

If user $U_{i}$ wants to change his/her password, $U_{i}$ inserts the smart card to the card reader and submits $I D_{i}$ and $P W_{i}^{\prime}$. The reader computes $z_{i}^{\prime}=y_{i} \oplus h\left(P W_{i}^{\prime}\right)$ and checks whether computed $z_{i}^{\prime}$ equals stored $z_{i}$. If equal, $U_{i}$ enters a new password $P W_{i}^{\text {new }}$. The card reader computes $z_{i}^{\text {new }}=y_{i} \oplus h\left(P W_{i}^{n e w}\right), x_{i}^{\text {new }}=x_{i} \oplus$
$g^{h\left(P W_{i}\right)} \oplus g^{h\left(P W_{i}^{n e w}\right)} \bmod p$ and stores $z_{i}^{\text {new }}, x_{i}^{\text {new }}$ instead of $z_{i}, x_{i}$ respectively in the memory of his/her smart card.

\section{SECURITY ANALYSIS OF TAYGI ET AL.'S SCHEME}

In this section, we will analyze the security of Taygi et al.'s [1] remote user authentication scheme in multi-server environment using smart card. To analyze the security weaknesses, it can be assumed that an attacker could obtain the secret values stored in the smart card by monitoring the power consumption [16][17] and intercepting messages communicating between the user and the server. Under this assumption, it will be discussed the various attacks, such as the user impersonation attack, server masquerading attack, collaboration attack between a valid user and a server, smart card stolen attack and password guessing attack on Taygi et al.'s [1] scheme. In addition, Taygi et al.'s [1] scheme does not provide mutual authentication between user and authentication server.

\subsection{User Impersonation Attack}

If an attacker can obtain the secret values $\left(x_{i}, y_{i}, z_{i}\right)$ from the user's smart card illegally by some means and intercept the message $\left\{I D_{i}, S I D_{j}, d_{i}, e_{i}, N_{1}\right\}$ in the login phase, the attacker can perform the user impersonation attack as the following steps:

Step 1: Attacker computes which is equal to $h\left(P W_{i}\right)=z_{i} \oplus y_{i}$

Step 2: The attacker generates a random nonce $N^{*}$ and compute $a_{i}=g^{y_{i}} \bmod p, b_{i}^{*}=a_{i}{ }^{y_{i} \times N^{*}} \bmod p, c_{i}^{*}=a_{i}{ }^{h\left(P W_{i}\right) \times N^{*}} \bmod p$, $d_{i}=g^{h\left(P W_{i}\right)} \bmod p$ and $Q_{j}=g^{h\left(S I D_{j},\left(x_{i} \oplus d_{i}\right)\right)} \bmod p, e_{i}^{*}=$ $\left(h\left(P W_{i}\right)+y_{i} \times h\left(I D_{i}, a_{i}, b_{i}^{*}, c_{i}^{*}, d_{i}, N^{*}, Q_{j}\right)\right) \bmod (p-1)$ then attacker sends $m s g=\left\{I D_{i}, S I D_{j}, d_{i}, e_{i}^{*}, N^{*}\right\}$ to the authenticated server $S_{j}$.

Step 3: After receiving the forged message $m s g, S_{j}$ checks the validity of $I D_{i}$ and compute $y_{i}=h\left(I D_{i}, h(d)\right), a_{i}=$ $g^{y_{i}} \bmod p, b_{i}^{*}=a_{i}{ }^{y_{i} \times N^{*}} \bmod p, c_{i}^{*}=d_{i}{ }^{y_{i} \times N^{*}} \bmod p$, $Q_{j}=S S_{j} \oplus h(d)$. Then verifies whether $g^{e_{i}^{*}}=d_{i} \times$ $a_{i}^{h\left(I D_{i}, a_{i}, b_{i}^{*}, c_{i}^{*}, d_{i}, N^{*}, Q_{j}\right)} \bmod p$ and $a_{i} e_{i}^{* \times N^{*}}=c_{i}^{*} \times$ $b_{i}^{*} h\left(I D_{i}, a_{i}, b_{i}^{*}, c_{i}^{*}, d_{i}, N^{*}, Q_{j}\right) \bmod p$. It can be shown that both are equal then authenticated server will be convinced the message sent from the legal user.

Step 4: After choosing a random nonce $N_{2}$, authenticated server $S_{j}$ makes the reply message $\left\{I D_{i}, X_{1}, X_{2}\right\}$ by computing $X_{1}$ $=y_{i} \oplus N^{*} \oplus N_{2}, X_{2}=Q_{j}{ }^{N_{2}} \bmod p$ in the authenticated phase.

\subsection{Server Masquerading Attack}

After obtaining the secret values $\left(x_{i}, y_{i}, z_{i}\right)$ from the user's smart card, further the attacker intercept the message $\left\{I D_{i}, S I D_{j}, d_{i}, e_{i}, N_{1}\right\}$ and $\left(X_{1}, X_{2}\right)$ in the login phase and authentication phase respectively, then the attacker can perform the Server masquerading attack as the following steps:

Step 1: The attacker computes $\left.Q_{j}=g^{h\left(S I D_{j},\left(x_{i} \oplus d_{i}\right)\right)} \bmod p\right)$. Then it chooses a random number $r$ and again compute $X_{1}=$ $y_{i} \oplus N_{1} \oplus r, X_{2}=Q_{j}{ }^{r} \bmod p$ and sends $\left\{I D_{i}, X_{1}, X_{2}\right\}$ to the user $U_{i}$.

Step 2: After receiving the forged message, user $U_{i}$ compute $r=$ $y_{i} \oplus X_{1} \oplus N_{1}, X_{2}^{\prime}=Q_{j}{ }^{r} \bmod p$ and verifies whether $X_{2}=X_{2}^{\prime}$. It can be easily verified that $X_{2}=X_{2}^{\prime}$.

Step 3: Then user $U_{i}$ makes the reply message $\left\{I D_{i}, X_{3}\right\}$ by computing $X_{3}=Q_{j}^{N_{1} \times r} \bmod p$. 


\subsection{Collaboration Attack between a Valid User and a Server}

In Taygi et al.'s [1] scheme a valid user $U_{i}$ can compute $h(x)$ $=x_{i} \oplus d_{i}$ where $d_{i}=g^{h\left(P W_{i}\right)} \bmod p$ and $x_{i}$ is stored in smart card of user $U_{i}$. Now let valid user $U_{i}$ provides this computed $h(x)$ to an another authenticated server $S_{m}$. Then $S_{m}$ can easily compute the secret key of another authenticated server $S_{j}$, by computing $S S_{j}=\left(g^{h\left(S I D_{j}, h(x)\right)} \bmod p\right) \oplus h(d)$, where $S I D_{j}$ is known from login message and $h(d)$ is known to all authenticated servers. Suppose another valid user $U_{k}$ is trying to communicate securely to authenticated server $S_{j}$. After sending a valid login message to $S_{j}$, another authenticated server $S_{m}$ can verify the user $U_{k}$ because $S_{m}$ can compute the secret key of $S_{j}$. If the server $S_{m}$ sends forge message $\left\{I D_{k}, X_{1 m}, X_{2 m}\right\}$ earlier than $S_{j}$ to the user $U_{k}$ by computing $X_{1 m}=y_{k} \oplus N_{1} \oplus N_{3}$, $X_{2 m}=Q_{j}{ }^{N_{3}} \bmod p$, where $N_{3}$ is randomly chosen by $S_{m}$. Then following scenario can be happened.

Step 1: User $U_{k}$ will accept the forge message which is send by $S_{m}$.

Step 2: At that time if another valid verification message $\left\{I D_{k}, X_{1}, X_{2}\right\}$ comes from $S_{j}$ by computing $X_{1}=y_{k} \oplus N_{1} \oplus$ $N_{2}, X_{2}=Q_{j}{ }^{N_{2}} \bmod p$ where $N_{2}$ is randomly chosen by $S_{j}$. The card reader will confuse which one will be accepted.

Step 3: If card reader choose forge message of $S_{m}$ and send $\left(I D_{k}, X_{3}\right)$ to $S_{j}$ by computing $X_{3}=Q_{j}^{N_{1} \times N_{3}} \bmod p . S_{j}$ will reject the valid user $U_{k}$ because $X_{3}=Q_{j}{ }^{N_{1} \times N_{3}} \bmod p$ and $X_{3}^{\prime}=Q_{j}{ }^{N_{1} \times N_{2}} \bmod p$ will not same.

\subsection{Password Guessing Attack}

After extracting the secret values $\left(x_{i}, y_{i}, z_{i}\right)$ from a legal user's smart card by some means, the attacker can easily find out $P W_{i}$ using following steps:

Step 1: An attacker computes $h\left(P W_{i}^{*}\right)$ and checks the correctness whether $h\left(P W_{i}^{*}\right)=z_{i} \oplus y_{i}$.

Step 2: An attacker repeats the above process until the correct password is obtained. After some guessing, an attacker can find out the correct password.

\subsection{Smart Card Stolen Attack}

Suppose a user $U_{i}$ either lost or stolen by an attacker of his/her smart card. After getting the smart card, the attacker can extract the secret information $\left\{x_{i}, y_{i}, z_{i}, p, g, h().\right\}$ from the user's smart card. We also assume that attacker store the $i-t h$ login message of the user $U_{i}$. As a result attacker can store the value $\left\{I D_{i}, S I D_{j}, d_{i}, e_{i}, N_{1}\right\}$. By using these secret information and stored parameter, attacker can create valid login message, described in user impersonation and server masquerading attack procedure in section 3 .

\subsection{Password Change Attack}

After obtaining secret values $\left(x_{i}, y_{i}, z_{i}\right)$ from the legal user's smart card, an attacker can easily find out $P W_{i}$ by performing the password guessing technique as describe in attack subsection 3.4. After getting correct $P W_{i}$, the attacker can change the valid user's password from the password change phase. So card reader will always reject the valid user in login phase.

\section{PROPOSED SCHEME}

In this section, we will propose the improvement of Taygi et al.'s [1] remote user authentication scheme in multi-server environment using smart card to overcome their weaknesses. It is assumed that Registration Center (RC) is a trusted authority.
We now describe the registration procedure of proposed scheme is as follows:

\subsection{Registration Phase}

This phase is divided into two sub-phases: Server Registration phase and User Registration phase.

\subsubsection{Server Registration Phase}

In this phase, $S_{j}$ selects $S I D_{j}$ and submits it to RC over a secure channel. After receiving the registration request from $S_{j}, \mathrm{RC}$ generate a random nonce $w_{j}$ for each server $(j=1$ to $\mathrm{m})$ and computes the server secret parameter $S S_{j}=h\left(S I D_{j}, h(x \|\right.$ $d)) \oplus h\left(x \| w_{j}\right)$ and sends $\left\{S S_{j}, h(d), h\left(x \| w_{j}\right)\right\}$ to $S_{j}$ over a secure channel.

\subsubsection{User Registration Phase}

$U_{i}$ selects $I D_{i}, P W_{i}$ and computes $B_{i}=g^{P W_{i} \times k} \bmod \mathrm{p}, I D U_{i}$ $=h\left(I D_{i} \| B_{i}\right)$ and submits $\left\{I D U_{i}, B_{i}, I D_{i}\right\}$ to $\mathrm{RC}$ over a secure channel. After receiving registration request from $U_{i}$, RC chooses a random nonce $n_{i}$ for each user $(i=1$ to $n)$ and computes $Y_{i}=h\left(h\left(I D U_{i}\right) \| h(d)\right), Z_{i}=h\left(h\left(I D U_{i}\right) \|\right.$ $\left.h\left(B_{i}\right)\right), K_{i}=h\left(B_{i}\right) \oplus h(x \| d) \oplus h\left(x \| n_{i}\right), D_{i}=Z_{i} \oplus h(x \|$ $\left.n_{i}\right), G_{i}=h\left(I D U_{i}\left\|Z_{i}\right\| h\left(x \| n_{i}\right) \| h(x \| d)\right), X$ $=g^{h(d)} \bmod p$ and issues a smart card for $U_{i}$ after storing $\left\{K_{i}, Y_{i}, G_{i}, D_{i}, X, p, g, h(\cdot)\right\}$ into memory of user's smart card. After getting smart card, user $U_{i}$ store $k$ into memory of smart card.

\subsection{Login Phase}

$U_{i}$ inserts the smart card into the card reader and submit $I D_{i}$ and $P W_{i}$. The card reader computes $B_{i}^{*}=g^{P W_{i} \times k} \bmod p, I D U_{i}^{*}=$ $h\left(I D_{i} \| B_{i}^{*}\right), Z_{i}^{*}=h\left(h\left(I D U_{i}^{*}\right) \| h\left(B_{i}^{*}\right)\right), R_{1}=Z_{i}^{*} \oplus D_{i}, R_{2}=$ $K_{i} \oplus h\left(B_{i}^{*}\right) \oplus R_{1}, G_{i}^{*}=h\left(I D U_{i}^{*}\left\|Z_{i}^{*}\right\| R_{1} \| R_{2}\right)$ and checks whether computed $G_{i}^{*}$ equals stored $G_{i}$. If true, card reader generates a random nonce $N_{1}$ and computes $C_{1}=B_{i}^{* N_{1}} \bmod p$, $C_{2}=X^{P W_{i} \times k \times N_{1}} \bmod p, Q_{i}=h\left(S I D_{j}, R_{2}\right) \oplus h\left(C_{2}\right), C_{3}=$ $h\left(I D U_{i}^{*}\right) \oplus h\left(C_{1} \| C_{2}\right) \oplus Q_{i}, L_{i}=h\left(h\left(I D U_{i}^{*}\right) \| Y_{i}\right), E_{i}=$ $\left(h\left(I D U_{i}^{*}\right)+Y_{i} \times h\left(L_{i}\left\|Q_{i}\right\| C_{2} \| h\left(I D U_{i}^{*}\right)\right) \bmod (p-1)\right.$ and sends the login request message $\left\{I D_{i}, S I D_{j}, Y_{i}, C_{1}, E_{i}, C_{3}\right\}$ to $S_{j}$.

In the following we prove the correctness of $G_{i}^{*}=G_{i}$ :

$$
\begin{aligned}
& G_{i}^{*}=h\left(I D U_{i}^{*}\left\|Z_{i}^{*}\right\| R_{1} \| R_{2}\right) \\
& R_{1}=Z_{i}^{*} \oplus D_{i}=Z_{i}^{*} \oplus Z_{i} \oplus h\left(x \| n_{i}\right)=h\left(x \| n_{i}\right), \text { since } Z_{i}^{*}= \\
& Z_{i} \\
& R_{2}=K_{i} \oplus h\left(B_{i}^{*}\right) \oplus R_{1} \\
& R_{2}=h\left(B_{i}\right) \oplus h(x \| d) \oplus h\left(x \| n_{i}\right) \oplus h\left(B_{i}^{*}\right) \oplus h\left(x \| n_{i}\right) \\
& R_{2}=h(x \| d), \text { Since } B_{i}=B_{i}^{*} \\
& G_{i}^{*}=h\left(I D U_{i}^{*}\left\|Z_{i}^{*}\right\| h\left(x \| n_{i}\right) \| h(x \| d)\right)=G_{i}, \text { since } I D U_{i}^{*} \\
& =I D U_{i} .
\end{aligned}
$$

\subsection{Authentication Phase}

After receiving the login request message $\left\{I D_{i}, S I D_{j}, Y_{i}, C_{1}\right.$, $\left.E_{i}, C_{3}\right\}, S_{j}$ first check the format of $I D_{i}$. If it is valid then computes $C_{2}^{*}=C_{1}{ }^{h(d)} \bmod p, Q_{i}^{*}=S S_{j} \oplus h\left(x \| w_{j}\right) \oplus h\left(C_{2}^{*}\right)$, $R_{3}=h\left(C_{1} \| C_{2}^{*}\right) \oplus Q_{i}^{*} \oplus C_{3}, Y_{i}^{*}=h\left(R_{3} \| h(d)\right)$ and checks whether computed $Y_{i}^{*}$ equals $Y_{i}$. If equals then computes $L_{i}^{*}=$ $h\left(R_{3} \| Y_{i}^{*}\right), T_{2}=h\left(L_{i}^{*}\left\|Q_{i}^{*}\right\| C_{2}^{*} \| R_{3}\right), E_{i}^{*}=R_{3}+Y_{i}^{*} \times$ $T_{2} \bmod (p-1)$ and checks whether $E_{i}^{*}$ equals $E_{i}$. If equals then proceed to mutual authentication Phase else reject user $U_{i}$. Further $S_{j}$ generates a random nonce $N_{2}$ and computes $A_{1}=$ $N_{2} \oplus Q_{i}^{*} \oplus R_{3}$, SKey $_{i j}=h\left(Q_{i}^{*}\left\|N_{2}\right\| L_{i}^{*}\right), A_{2}=h\left(N_{2} \|\right.$ $\left.S K e y_{i j}\right)$. Then sends $\left\{A_{1}, A_{2}\right\}$ to user $U_{i}$. After receiving it, $U_{i}$ 
computes $N_{2}^{*}=A_{1} \oplus Q_{i} \oplus h\left(I D U_{i}^{*}\right), S K e y_{i j}^{*}=h\left(Q_{i}\left\|N_{2}\right\|\right.$ $\left.L_{i}\right), A_{2}^{*}=h\left(N_{2}^{*} \| S K e y_{i j}^{*}\right)$ and checks $A_{2}^{*}$ equals $A_{2}$. If equals then mutual authentication is achieved and both the parties agree with a common shared session key $S K e y_{i j}$ for securing future data communications.

In the following we prove the correctness of $Y_{i}^{*}=Y_{i}, E_{i}^{*}=E_{i}$ and $A_{2}^{*}=A_{2}$.

$$
\begin{aligned}
& \text { Correctness of } Y_{i}^{*}=Y_{i}: \\
& Y_{i}^{*}=h\left(R_{3} \| h(d)\right) \\
& R_{3}=h\left(C_{1} \| C_{2}^{*}\right) \oplus Q_{i}^{*} \oplus C_{3} \\
& R_{3}=h\left(C_{1} \| C_{2}^{*}\right) \oplus Q_{i}^{*} \oplus h\left(I D U_{i}^{*}\right) \oplus h\left(C_{1} \| C_{2}\right) \oplus Q_{i} \\
& R_{3}=h\left(I D U_{i}^{*}\right), \text { since } C_{2}^{*}=C_{2} \text { and } Q_{i}^{*}=Q_{i} \\
& Y_{i}^{*}=h\left(I D U_{i}^{*} \| h(d)\right)=Y_{i}, \text { Since } I D U_{i}^{*}=I D U_{i}
\end{aligned}
$$

Correctness of $E_{i}^{*}=E_{i}$ :

$$
\begin{aligned}
& E_{i}^{*}=R_{3}+Y_{i}^{*} \times T_{2} \bmod (p-1) \\
& E_{i}^{*}=h\left(I D U_{i}^{*}\right)+Y_{i} \times h\left(L_{i}^{*}\left\|Q_{i}^{*}\right\| C_{2}^{*} \| h\left(I D U_{i}\right)\right) \\
& E_{i}^{*}=E_{i}, \text { Since } L_{i}^{*}=L_{i}, Q_{i}^{*}=Q_{i} \text { and } C_{2}^{*}=C_{2} .
\end{aligned}
$$

Correctness of $A_{2}^{*}=A_{2}$ :

$$
\begin{aligned}
& A_{2}^{*}=h\left(N_{2}^{*} \| S K e y_{i j}^{*}\right) \\
& N_{2}^{*}=A_{1} \oplus Q_{i} \oplus h\left(I D U_{i}^{*}\right) \\
& N_{2}^{*}=N_{2} \oplus Q_{i}^{*} \oplus R_{3} \oplus Q_{i} \oplus h\left(I D U_{i}^{*}\right) \\
& N_{2}^{*}=N_{2}, \text { since } Q_{i}^{*}=Q_{i} \text { and } R_{3}=h\left(I D U_{i}^{*}\right) \\
& S K e y_{i j}^{*}=S K e y_{i j}, \text { since } N_{2}^{*}=N_{2} \\
& A_{2}^{*}=h\left(N_{2} \| S K e y_{i j}\right) \\
& A_{2}^{*}=A_{2}, \text { since } S K e y_{i j}^{*}=S K e y_{i j} .
\end{aligned}
$$

\subsection{Password Change Phase}

This phase is invoked when $U_{i}$ wants to change the password. $U_{i}$ inserts the smart card into the card reader and submits $I D_{i}$ and $P W_{i}$. Then card reader computes $B_{i}^{*}=g^{P W_{i} \times k} \bmod \mathrm{p}, I D U_{i}^{*}$ $=h\left(I D_{i} \| B_{i}\right), Z_{i}^{*}=h\left(h\left(I D U_{i}^{*}\right) \| h\left(B_{i}^{*}\right)\right), R_{4}=Z_{i}^{*} \oplus D_{i}$, $R_{5}=K_{i} \oplus h\left(B_{i}^{*}\right) \oplus R_{4}, G_{i}^{*}=h\left(I D U_{i}^{*}\left\|Z_{i}^{*}\right\| R_{4} \| R_{5}\right)$ and checks whether $G_{i}^{*}$ equals stored $G_{i}$. If equals, $U_{i}$ enters a new password $P W_{i}^{\text {new }}$. Then card reader computes $B_{i}^{\text {new }}=$ $g^{P W_{i}^{n e w} \times k} \bmod \mathrm{p}, Z_{i}^{\text {new }}=h\left(h\left(I D U_{i}^{*}\right) \| h\left(B_{i}^{\text {new }}\right)\right), K_{i}^{\text {new }}=$ $h\left(B_{i}^{\text {new }}\right) \oplus R_{4} \oplus R_{5}, D_{i}^{\text {new }}=Z_{i}^{\text {new }} \oplus R_{4}, G_{i}^{\text {new }}=h\left(I D U_{i}^{*} \|\right.$ $\left.Z_{i}^{\text {new }}\left\|R_{4}\right\| R_{5}\right)$ and stores $K_{i}^{\text {new }}, D_{i}^{\text {new }}$ and $G_{i}^{\text {new }}$ instead of $K_{i}, D_{i}$ and $G_{i}$ respectively in the memory of smart card. Thus $U_{i}$ can change the password without taking any assistance from $S_{j}$.

\section{CRYPTANALYSIS OF PROPOSED SCHEME}

This section describes cryptanalysis of proposed scheme. In this paper, to analyze the security analysis of proposed scheme, it can be assumed that an attacker could obtain the secret values stored in the smart card by monitoring the power consumption [16][17] and intercept messages communicating between the user and the server. Also it can be assumed that an attacker may possess the capabilities to thwart the security scheme.

\subsection{User Impersonation Attack}

To impersonate as a legitimate user, an attacker attempts to make a forged login request message which can be authenticated to a server. However, the attacker can not impersonate as the legitimate user by forging the login request message even if the attacker can extract the secret values $\left\{K_{i}, Y_{i}, G_{i}, D_{i}, X, k\right\}$ stored in the user's smart card, because the attacker can not compute the valid login request message $\left\{E_{i}, C_{1}, C_{3}\right\}$ without knowing the secret password $P W_{i}$ of valid user $U_{i}$. In proposed scheme if the attacker wants to get the secret parameter $P W_{i}$, he/she must have to solve the inversion of cryptographic hash function which is computationally hard. So proposed scheme is secured against user impersonation attack.

\subsection{Server Masquerading Attack}

To masquerade as a legitimate server, an attacker attempts to make the forged reply message which can be masqueraded to the user when receiving the users login request message. However, the attacker can not masquerade as the server by forging the reply message, because it is hard to compute $\left\{A_{1}, A_{2}\right\}$ by an attacker without knowing the secret value $h(d)$ and $h\left(x \| w_{j}\right)$ kept by the server. Hence the attacker can not masquerade as the legitimate server to the user by launching the server masquerading attack.

\subsection{Collaboration Attack between a Valid User and a Server}

In proposed scheme to perform collaboration attack between a valid user and a server, another authenticated server $S_{m}$ needs to compute the secret key of $S_{j}$. To compute the secret key of $S_{j}, S_{m}$ needs to compute $h\left(x \quad \| \quad w_{j}\right)$ from the communicating message between the user $U_{i}$ and the server $S_{j}$. But the communicating message between the user and server are independent of $h\left(x \| w_{j}\right)$. Hence it is hard to compute the secret key $S S_{j}$ of the server $S_{j}$ by another server $S_{m}$. So proposed scheme is secured against the collaboration attack between a valid user and a server.

\subsection{Password Guessing Attack}

After getting secret values $\left\{K_{i}, Y_{i}, G_{i}, D_{i}, X, k\right\}$ stored in the user $U_{i}$ smart card, the attacker attempts to derive the user $U_{i}$ 's password $P W_{i}$ using $K_{i}=h\left(B_{i}\right) \oplus h(x \| d) \oplus h\left(x \| n_{i}\right)$, $G_{i}$ and $D_{i}$ in the user registration phase. To guess the user's password, attacker needs to compute $B_{i}$ from $K_{i}, G_{i}$ and $D_{i}$ but it is not possible. So proposed scheme is secured against password guessing attack.

\subsection{Smart Card Stolen Attack}

It can be assumed that the user $U_{i}$ has either lost his/her smart card or stolen by an attacker. After getting the smart card, an attacker can extract the secret information $\left\{K_{i}, Y_{i}, G_{i}, D_{i}, X, k\right\}$ from the smart card. Also can be assumed that attacker stores the $i-t h$ login message $\left\{S I D_{j}, Y_{i}, C_{1}, E_{i}, C_{3}\right\}$ of the user $U_{i}$. After getting all these parameter that is login message and smart card parameter, it is hard to derive $P W_{i}$, server secret information $h(d)$ and $h\left(x \| w_{j}\right)$ by the attacker. So attacker can not create the valid login message. As a result proposed scheme is secured against smart card stolen attack.

\subsection{Insider Attack}

Generally, many user uses same password for their convenience of remembering and easy of use whenever required. However if the system manager or privileged insider of the server knows user's password, he/she may try to access user's $U_{i}$ other accounts in other server. But in proposed scheme, the system manager or privileged insider of the server can not derive user's password $P W_{i}$ because user have submitted $h\left(B_{i}\right)=$ $g^{P W_{i} \times k}$ mod $p$ instead of $P W_{i}$. To get correct $P W_{i}$, system manager must have to solve Diffie-Hellman problem [18] which is computationally hard. So proposed scheme resists insider attack. 


\subsection{Password Change Attack}

As described in attack 5.4 and attack 5.5 in section 5, proposed scheme can withstand the Password guessing attack and smart card stolen attack. To perform password change attack, an attacker have to provide correct $I D_{i}$ and $P W_{i}$ to the card reader. But in proposed scheme, there is no way to get correct $P W_{i}$ to the attacker. So proposed scheme is secured against password change attack.

\section{PERFORMANCE ANALYSIS OF PROPOSED SCHEME}

In this section we evaluated the performance of proposed scheme with Taygi et al.'s [1] scheme. We have compare login and authentication phases of proposed scheme with Taygi et al.'s [1] scheme because these phases are used frequently. Table 1 shows the computation over head and Table 2 shows the communication cost and storage cost of proposed scheme and Taygi et al.'s [1] scheme. In Table $1, T_{h}$ is the time required for hashing operation and $T_{e}$ is the time required for exponentiation operation and $T_{m}$ is the time required for multiplication operation. Though, proposed scheme resists different possible attacks of Taygi et al.'s [1] scheme, in spite of proposed scheme provides better computation cost than the related scheme because of exponentiation and multiplication operation. In proposed scheme, only four (4) exponentiation and five (5) multiplication are used whereas Taygi et al.'s [1] scheme requires sixteen (16) exponentiation and eight (8) multiplication operations.

It can be reasonably assumed that the length of $I D_{i}, P W_{i}$ and $S I D_{j}$ is 64 bits each and $h(\cdot)$, random nonce returns 128 bits and length of $g, p$ are 1024 bits each. The communication cost (capacity of transmitting message) of proposed scheme and Taygi et al.'s [1] scheme is 2688 bits $=(64+64+128+1024+$ $1024+128+128+128)$ and 4608 bits $=(64+64+1024+$ $1024+128+64+128+1024+64+1024)$ respectively. Also the storage cost (stored into the memory of smart card) takes almost same bits of proposed scheme and their scheme that is 3840 bits, 3456 bits respectively. Table 3 shows that their scheme is insecure against different possible attacks. Further proposed scheme provides strong authentication against different attacks described in section 5. After resisting all possible attacks of related scheme, proposed scheme provides low computational and low communication cost than their scheme. Hence proposed scheme is more efficient and secure than Taygi et al.'s [1] scheme.

Table 1. comparison of computational cost of proposed scheme with related scheme

\begin{tabular}{|c|c|c|}
\hline $\begin{array}{c}\text { Scheme } \Rightarrow \\
\text { Phase } \Downarrow\end{array}$ & Taygi et al.'s $\llbracket 1$ & Our \\
\hline Login Phase & $3 T_{h}+5 T_{e}+3 T_{m}$ & $10 T_{h}+3 T_{e}+4 T_{m}$ \\
\hline Authentication Phase & $4 T_{h}+11 T_{e}+5 T_{m}$ & $9 T_{h}+1 T_{e}+1 T_{m}$ \\
\hline
\end{tabular}

Table 2. comparison of communication and storage cost of proposed scheme with related scheme

\begin{tabular}{|c|c|c|}
\hline $\begin{array}{c}\text { Scheme } \Rightarrow \\
\text { Item } \Downarrow\end{array}$ & Taygi et al.'s [1] & Our \\
\hline Communication Cost & 4608 bits & 2688 bits \\
\hline Storage Cost & 3456 bits & 3840 bits \\
\hline
\end{tabular}

Table 3. attack comparison of proposed scheme with related scheme

\begin{tabular}{|l|c|c|}
\hline \multicolumn{1}{|c|}{$\begin{array}{c}\text { Scheme } \Rightarrow \\
\text { Attack } \Downarrow\end{array}$} & Taygi et al.'s [1] & Our \\
\hline User Impersonation Attack & Yes & No \\
\hline Server Masquerading Attack & Yes & No \\
\hline $\begin{array}{l}\text { Collaboration Attack between } \\
\text { a Valid User and a Server }\end{array}$ & Yes & No \\
\hline Password Guessing Attack & Yes & No \\
\hline Smart Card Stolen Attack & Yes & No \\
\hline Password Change Attack & Yes & No \\
\hline Mutual Authentication & No & Yes \\
\hline
\end{tabular}

\section{(1) Mutual Authentication}

As described the attack procedure in section 5, proposed scheme can withstand the user impersonation attack and the server masquerading attack, consequently the proposed scheme provides mutual authentication between the user and the remote server. Namely, even if the attacker can extract the secret values $\left\{K_{i}, Y_{i}, G_{i}, D_{i}, X, k\right\}$ stored in the users smart card, the user can be authenticated to the server and vice versa. Because the attacker can not make the login request message $\left\{S I D_{j}, Y_{i}, C_{1}, E_{i}, C_{3}\right\}$ and the reply message $\left\{A_{1}, A_{2}\right\}$ without knowing the secret value $P W_{i}$ of user $U_{i}$ and the secret values $S S_{i}, h(d)$ of the server $S_{j}$ respectively.

(2) Single Registration

In proposed scheme, if a user register to registration server once then the user can access all the registered servers.

\section{(3) No Verification Table}

None of the registered servers need to maintain a verification table.

(4) Securely Password Change Without Taking Help From Server and RC

A valid user can change the password freely and securely without taking any help from the servers or registration center. As the card reader first verifies the old password in the password change phase, so unauthorized users cannot change the authorized users password even if they get the valid user's smart card.

\section{(5) Early Wrong Password Detection}

If the user $U_{i}$ inputs a wrong password by mistake in password change phase, it will be quickly detected by the card reader itself since card reader computes $B_{i}^{*}$ $=g^{P W_{i} \times k} \bmod p, I D U_{i}^{*}=h\left(I D_{i} \| B_{i}^{*}\right), Z_{i}^{*} \stackrel{i}{=}$ $h\left(h\left(I D U_{i}^{*}\right) \| h\left(B_{i}^{*}\right)\right), R_{1}=Z_{i}^{*} \oplus D_{i}, R_{2}=K_{i} \oplus h\left(B_{i}^{*}\right) \oplus$ $R_{1}, G_{i}^{*}=h\left(I D U_{i}^{*}\left\|Z_{i}^{*}\right\| R_{1} \| R_{2}\right)$ and checks whether $G_{i}^{*}$ equals $G_{i}$. Hence proposed scheme provides early wrong password detection.

\section{(6) Server's Unique Secret Parameter}

In proposed scheme, each server has unique secret parameter $S S_{j}=h\left(S I D_{j}, h(x \| d)\right) \oplus h\left(x \| w_{j}\right)$ used to authenticate the user. Hence there is no need to store the secret parameter of all the servers in the memory of smart card.

(7) Mutual Authentication and Session Key Agreement Without Help of RC

Any valid users and valid servers can authenticate each other and then agree on a session key without any support from 
the registration center. The generated session key $S K e y_{i j}=$ $h\left(Q_{i}^{*}\left\|N_{2}\right\| L_{i}^{*}\right)$ will be different for each login session.

\section{(8) Solves Time Synchronization Problem}

The proposed scheme uses randomly generated nonce $N_{1}$ and $N_{2}$ instead of time stamps to avoid time synchronization problem.

\section{CONCLUSION}

This paper shows that Taygi et al.'s [1] scheme suffers from different attacks. To over come these weaknesses a proposed scheme is given in this paper. Further, the proposed scheme is more efficient in terms of computational and communication cost than that of Taygi et al.'s [1] scheme. In addition, proposed scheme provides mutual authentication between user and authentication server and also user can change his/her password freely without help of registration server.

It is shown that the proposed scheme provides strong security protocol based on the user's password. For the better security, biometric feature as well as password can be incorporate in the protocol.

\section{REFERENCES}

[1] J.K. Tyagi, A.K. Srivastava and P.S. Patwal, "Remote user authentication scheme in multi-server environment using smart card", International Journal of Computer Applications (0975 8887), vol. 57, no. 12, pp. 1-5, November 2012.

[2] Li, L., Lin, I., Hwang, M. S., "A remote password authentication scheme for multi-server architecture using neural networks", IEEE Transaction on Neural Networks, vol. 12, pp. 1498-1504, 2001.

[3] Lin, I. C., Hwang, M. S., Li, L. H., "A new remote user authentication scheme for multi-server architecture", Future Generation Computer Systems, vol. 19, pp. 13-22, 2003.

[4] Elgamal, T., "A public key cryptosystem and a signature scheme based on discrete logarithms", IEEE Transactions on Information Theory, vol. 31(4), pp. 469-472, 1985.

[5] Cao, X., Zhong, S., "Breaking a remote user authentication scheme for multi-server architecture", IEEE Communications Letters, vol. 10, pp. 580-581, 2006.

[6] Juang, W. S., "Efficient password authenticated key agreement using smart cards", Computers and Security vol. 23, pp. 167-173, 2004.
[7] Ku, W. C., Chuang, H. M., Chiang, M. H. and Chang, K. T., "Weaknesses of a multi-server password authenticated key agreement scheme", In Proceedings of the 2005 National computer Symposium, vol. 138, pp. 1-5, 2005.

[8] Chang, C. C., Lee, J. S., "An efficient and secure multi-server password authentication scheme using smart cards", In Proceedings of the International Conference on Cyberworlds, pp. 417-422, 2004.

[9] Liao, Y. P., Wang, S. S., "A secure dynamic ID based remote user authentication scheme for multi-server environment", Computer Standards and Interfaces, vol. 31, pp. 24-29, 2009.

[10] Chen, T. Y., Hwang, M. S., Lee, C. C. and Jan, J. K., "Cryptanalysis of a secure dynamic ID based remote user authentication scheme for multi-server environment", In Proceedings of the 4th International Conference on Innovative Computing, Information and Control, pp. 725728, 2009.

[11] Hsiang, C., Shih, W. K., "Improvement of the secure dynamic ID based remote user authentication scheme for multi-server environment", Computer Standards and Interfaces, vol. 31, pp. 1118-1123, 2009.

[12] Sood, S. K., Sarje, A. K., Singh, K., "A secure dynamic identity based authentication protocol for multiserver architecture", Journal of Network and Computer Applications, vol. 34, pp. 609-618, 2011.

[13] Tsai, J. L., "Efficient multi-server authentication scheme based on one-way hash function without verification table", Computers and Security, vol. 27, pp. 115-121, 2008.

[14] Zhu, H, Liu, T. and Liu, J., "Robust and simple multiserver authentication protocol without verification table", In Proceedings of the 9th International Conference on Hybrid Intelligent Systems, pp. 13-22, 2009.

[15] Denning, Dorothy E, Sacco, Giovanni Maria, "Timestamps in key distributed protocols", Communication of the ACM, vol. 24, pp. 533-535, 1981.

[16] P. Kocher, J. Jaffe, and B. Jun, "Differential power analysis", Proceedings of Advances in Cryptology, pp. 388-397, 1999.

[17] T. S. Messerges, E. A. Dabbish, and R. H. Sloan, "Examining smart-card security under the threat of power analysis attacks, IEEE Transactions on Computers, vol. 51, no. 5, pp. 541-552, 2002.

[18] Whitfield Diffie and Martin E. Hellman, "New Directions in Cryptography". IEEE Transactions on Information Theory, vol. IT-22 (6), pp. 644-654, November 1976. 